\title{
Towards a multi-sensor system for the evaluation of aseptic processes employing hydrogen peroxide vapour $\left(\mathrm{H}_{2} \mathrm{O}_{2}\right)$
}

\author{
Steffen Reisert ${ }^{1}$, Hanno Geissler ${ }^{2}$, Rudolf Flörke ${ }^{2}$, Niko Näther ${ }^{1}$, Patrick Wagner $^{3}$, \\ and Michael J. Schöning ${ }^{*, 1}$ \\ ${ }^{1}$ Institute of Nano- and Biotechnologies, Aachen University of Applied Sciences, 52428 Jülich, Germany \\ ${ }^{2}$ SIG Combibloc Systems GmbH, 52441 Linnich, Germany \\ ${ }^{3}$ Institute for Materials Research, Hasselt University, 3590 Diepenbeek, Belgium
}

Received 6 October 2010, revised 6 December 2010, accepted 6 December 2010

Published online 10 May 2011

Keywords aseptic processes, Bacillus subtilis, hydrogen peroxide, multi-sensor

*Corresponding author: e-mail schoening@fh-aachen.de, Phone: +49 2416009 53215, Fax: +49 241600953235

The presented work focuses on the development of a multisensor system for the evaluation of aseptic processes employing hydrogen peroxide vapour. In a first step, selected commercially available gas sensors have been investigated on crosssensitivity towards hydrogen peroxide vapour and humidity. An MOX (TGS 816, Figaro) and a solid-electrolyte gas sensor (SO-A0-250, Electrovac) ( $\lambda$-probe) have shown good characteristics in terms of sensitivity towards HPV, considering also reproducibility, long-term drift and stability. Further, the sterilization effect of HPV has been investigated by means of the microbial reduction test. A correlation between the microbial reduction of Bacillus subtilis spores and the sensor response in hydrogen peroxide atmosphere has been established. Based on this correlation the microbial reduction may be estimated by means of the sensor output over a wide range of parameters.
1 Introduction The aseptic filling of pre-sterilized foodstuff has reached a high level of technical sophistication and is growing in importance [1]. Due to its strong antimicrobial properties and environmental compatibility, hydrogen peroxide vapour is the method of choice for the inactivation of microorganisms such as bacteria, fungi, viruses and highly resistant spores [2-7]. The microbial inactivation process by hydrogen peroxide vapour is carried out at temperatures above $200{ }^{\circ} \mathrm{C}$ and concentrations of $\mathrm{H}_{2} \mathrm{O}_{2}$ up to $10 \% \mathrm{v} / \mathrm{v}$. The inactivation of microorganisms by hydrogen peroxide has been the subject of various studies. It was shown that the microbial inactivation favourably occurs at high concentrations of hydrogen peroxide and elevated gas temperatures [7-12].

The effectiveness of aseptic sterilization processes by hydrogen peroxide vapour may be determined by the socalled challenge test that requires artificial inoculation of packaging materials with microorganisms under controlled conditions in order to obtain statistically significant test results allowing objective comparison [13,14]. Even though this represents a well established and reliable method, it is to some degree unfavourable as probes require a lot of preparation and an inoculation time between $24 \mathrm{~h}$ and a few days.

Several attempts have been made to characterize sterilization processes by monitoring the influence parameters, as such the hydrogen peroxide concentration or temperature [15-21]. However, monitoring of one single parameter is often not sufficient for an accurate evaluation of a sterilization process, since there is an interaction between the various influential parameters, such as temperature, pressure, gas flow and humidity, which can have a decisive effect on the inactivation of microorganisms. One promising approach for the evaluation of multi-component chemical media and dynamic processes relies on the use of multisensor systems [22]. Concerning such multi-sensor systems, multi-component chemical media could be described not by a sum of the individual components (or corresponding response from the specific sensors) but by some abstract representation - a chemical image - a virtual fingerprint with a set of parameters intrinsical for a given multi-component medium [23]. 
In this work, an approach for the development of a multisensor system for the evaluation of aseptic processes employing hydrogen peroxide vapour is presented. Chemical images of selected gas sensors in hydrogen peroxide atmosphere are contrasted with the results of microbiological count reduction tests (CRTs) of Bacillus subtilis. A correlation between the effectiveness of aseptic sterilization processes and the response of the selected gas sensors could be established via the hydrogen peroxide concentration.

\section{Experimental}

2.1 Test equipment To perform the microbiological tests and investigate the sensor properties, an experimental set-up in laboratory scale, similar to industrial apparatuses, has been used for the generation of hydrogen peroxide vapour. The set-up provides a carrier gas stream (compressed air), which is fed with aqueous hydrogen peroxide solution and/or water by two dosing pumps. In a subsequent heater the aerosol is evaporated. An integrated temperature regulation allows controlling the outlet temperature of the evaporated medium, which is equally dispensed out of four gas nozzles. With this experimental set-up, a wide range of gas compositions, flow rates and gas temperatures can be generated. A more detailed description of the experimental set-up can be found elsewhere [16].

For the sensor characterization and microbiological testing a set of parameters, which represents common settings for industrial sterilization processes, has been chosen. All measurements have been carried out at a gas flow rate of $10 \mathrm{~m}^{3} / \mathrm{h}$ and a gas temperature of $270{ }^{\circ} \mathrm{C}$, but different levels of hydrogen peroxide concentration in gaseous phase. Therefore, on one hand, different amounts

Table 1 Parameter for the sensor calibration of measurement 1. Constant hydrogen peroxide-water ratio.

\begin{tabular}{llllll}
\hline dosage $(\mu 1 / \mathrm{s})^{\mathrm{a}}$ & 250 & 500 & 750 & 1000 & 1100 \\
\hline $\mathrm{C}\left[\mathrm{H}_{2} \mathrm{O}_{2}\right](\% \mathrm{v} / \mathrm{v})$ & 2.3 & 4.1 & 5.7 & 6.9 & 7.4 \\
$\mathrm{C}\left[\mathrm{H}_{2} \mathrm{O}\right](\% \mathrm{v} / \mathrm{v})$ & 7.9 & 14.3 & 19.7 & 24.2 & 25.8 \\
$\mathrm{C}\left[\mathrm{H}_{2} \mathrm{O}_{2}\right] / \mathrm{C}\left[\mathrm{H}_{2} \mathrm{O}\right]$ & 0.285 & 0.285 & 0.285 & 0.285 & 0.285 \\
\hline
\end{tabular}

${ }^{a}$ Measurements have been carried out at a constant volume flow of $10 \mathrm{~m}^{3} / \mathrm{h}$, a constant gas temperature of $270{ }^{\circ} \mathrm{C}$ and varying dosage of a $35 \% \mathrm{w} / \mathrm{w}$ technical grade aqueous hydrogen peroxide solution.

Table 2 Parameter for the sensor calibration of measurement 2. Varying hydrogen peroxide-water ratio.

\begin{tabular}{lccc}
\hline $\mathrm{C}_{\mathrm{sol}}\left[\mathrm{H}_{2} \mathrm{O}_{2}\right](\% \mathrm{w} / \mathrm{w})^{\mathrm{a}}$ & 15 & 25 & 35 \\
$\mathrm{C}\left[\mathrm{H}_{2} \mathrm{O}_{2}\right](\% \mathrm{v} / \mathrm{v})$ & 2.2 & 3.9 & 5.6 \\
$\mathrm{C}\left[\mathrm{H}_{2} \mathrm{O}\right](\% \mathrm{v} / \mathrm{v})$ & 23.8 & 21.8 & 19.7 \\
$\mathrm{C}\left[\mathrm{H}_{2} \mathrm{O}_{2}\right] / \mathrm{C}\left[\mathrm{H}_{2} \mathrm{O}\right]$ & 0.092 & 0.176 & 0.285
\end{tabular}

${ }^{a}$ Measurements have been carried out at a constant volume flow of $10 \mathrm{~m}^{3} / \mathrm{h}$, a constant gas temperature of $270^{\circ} \mathrm{C}$ and varying dilutions of a technical grade aqueous hydrogen peroxide solution $\left(\mathrm{C}_{\mathrm{sol}}\left[\mathrm{H}_{2} \mathrm{O}_{2}\right]\right)$ at a constant dosage of $750 \mu \mathrm{l} / \mathrm{s}$. of a technical grade aqueous hydrogen peroxide solution of $35 \% \mathrm{w} / \mathrm{w}$ have been fed to the carrier gas stream. On the other hand, measurements have been performed at a constant dosage but different dilutions of the hydrogen peroxide solution. While at the first experiment the ratio between hydrogen peroxide and water in gaseous phase was kept constant, it is different for the latter experiment. In this way, the influence of humidity on the sensor signal and microbial reduction shall be investigated. The highest concentration of hydrogen peroxide in gaseous phase was achieved at a gas flow of $10 \mathrm{~m}^{3} / \mathrm{h}$ and the maximum possible dosage of $1100 \mu \mathrm{l} / \mathrm{s}$ of a $35 \% \mathrm{w} / \mathrm{w}$ technical grade hydrogen peroxide solution. Four series of measurement have been carried out, each time two measurements for the sensor calibration and the microbiological tests, respectively. The parameters of the four series of measurement are listed in Tables 1-4. (Constant hydrogen peroxide - water ratio, varying hydrogen peroxide - water ratio).

2.2 Gas sensors Two types of commercially available gas sensors have been investigated on cross-sensitivity towards hydrogen peroxide vapour: The metal-oxidesemiconductor gas sensor (MOX) TGS 816 from Figaro Engineering (Japan) and the amperometric solid-electrolyte sensor ( $\lambda$-probe) SO-A0-250 from Electrovac AG (Austria). Hydrogen peroxide has strong oxidizing properties. Semiconductor gas sensors are known for their high sensitivity to reducing and oxidizing gases, while in case of the solid-electrolyte sensor a reaction to oxygen, originated from the decomposition of hydrogen peroxide on either the heater surface or even the sensor surface itself, is expected. The TGS 816 was heated with a constant voltage of $5 \mathrm{~V}$, while the SO-A0-250 was operated in a temperature-

Table 3 Parameter of the microbiological measurement 1. Constant hydrogen peroxide-water ratio.

\begin{tabular}{lllll}
\hline dosage $(\mu \mathrm{l} / \mathrm{s})^{\mathrm{a}}$ & 300 & 500 & 600 & 900 \\
\hline $\mathrm{C}\left[\mathrm{H}_{2} \mathrm{O}_{2}\right](\% \mathrm{v} / \mathrm{v})$ & 2.6 & 4.1 & 4.7 & 6.4 \\
$\mathrm{C}\left[\mathrm{H}_{2} \mathrm{O}\right](\% \mathrm{v} / \mathrm{v})$ & 9.3 & 14.3 & 16.6 & 22.5 \\
$\mathrm{C}\left[\mathrm{H}_{2} \mathrm{O}_{2}\right] / \mathrm{C}\left[\mathrm{H}_{2} \mathrm{O}\right]$ & 0.285 & 0.285 & 0.285 & 0.285 \\
\hline
\end{tabular}

${ }^{a}$ Measurements have been carried out at a constant volume flow of $10 \mathrm{~m}^{3} / \mathrm{h}$, a constant gas temperature of $270{ }^{\circ} \mathrm{C}$ and varying dosage of a $35 \% \mathrm{w} / \mathrm{w}$ technical grade aqueous hydrogen peroxide solution. 
controlled mode at $533{ }^{\circ} \mathrm{C}$ according to the manufacturer's data sheet. Both sensors were placed directly in the gas stream, ca. $5 \mathrm{~cm}$ below the outlet of the gas nozzle.

2.3 Microbiological tests Microbiological tests have been performed according to the CRT [13]. It is a well established method for testing the microbiological effectiveness of aseptic sterilization processes. By making use of the CRT, the test items (in this work alumina strips have been used) are inoculated with test microorganisms and passed through the sterilization process. In doing so, the number of viable spores is determined before and after the sterilization and from the difference in microorganism counts the logarithmic count reduction (log-rate) is determined in accordance to the following formula:

$$
\log \text {-rate }=\log (\text { initial count })-\log (\text { final count }) .
$$

As test organism, spores of $B$. subtilis have been used. It is the test organism of choice regarding the sterilization by means of hydrogen peroxide. The test strips have been prepared by pipetting one drop of a spore suspension (spores of B. subtilis in $70 \%$ alcohol) on an alumina strip and were dried at room temperature in germ-free environment. The average initial count of spores on the test strips was $\approx 8 \times 10^{6}$. The test strips were exposed to the sterilization medium for an effective time of $500 \mathrm{~ms}$ (common rate of a sterilization/filling cycle of aseptic carton packages in a filling line). For each setting, a number of five samples has been taken and the number of viable spores has been determined. Therefore, the spores have been removed from the test strips in $10 \mathrm{ml}$ lactated Ringer's solution in an ultrasonic bath. Subsequently, different dilutions of each sample have been prepared. Of each dilution, $1 \mathrm{ml}$ was plated on nutrient agar and incubated at $37^{\circ} \mathrm{C}$ for $24 \mathrm{~h}$. After incubation, the number of colony forming units (CFU), whereby each of the CFU is originated from one viable spore, was determined and the resulting average log-rate of five samples was calculated. It is necessary to prepare different dilutions of each sample in order to obtain a countable number $(<300)$ of CFU on the agar plate. In this work, dilutions up to $1: 10^{4}$ have been prepared. From the degree of dilution and amount of solution plated on the agar, it is possible to count back the number of viable spores of the initial sample. A more detailed protocol of the inoculation procedure and test method can be found in Ref. [13].

\section{Results and discussion}

3.1 Calibration of the gas sensors To investigate the cross-sensitivity of the gas sensors towards hydrogen peroxide vapour, two series of measurement have been carried out as described in Section 2.2.

3.2 TGS 816 Figure 1 shows the results of the TGS 816 obtained from measurements 1 and 2, plotted against the assumed hydrogen peroxide concentration calculated from the mixer flow ratios. The measured data is depicted as

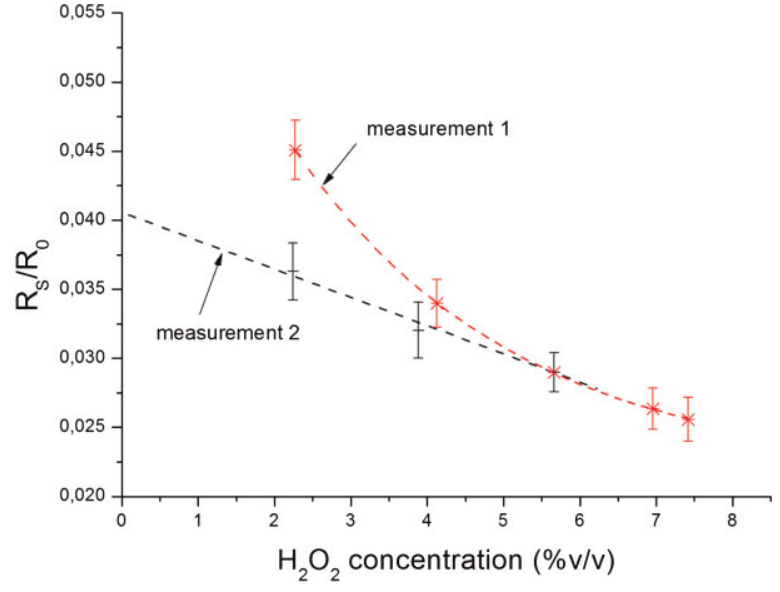

Figure 1 (online colour at: www.pss-a.com) Normalized sensor signals of the $\mathrm{SnO}_{2}$ semiconductor gas sensor TGS 816 at different concentrations of hydrogen peroxide operated with a constant heating voltage of $5 \mathrm{~V}$.

relative resistance values $R_{\mathrm{S}} / R_{0} . R_{0}$ was determined in the hot gas stream at a constant gas flow of $10 \mathrm{~m}^{3} / \mathrm{h}$ and a gas temperature of $270^{\circ} \mathrm{C}$, without the presence of hydrogen peroxide.

From the measurement values diagrammed in Fig. 1 it can be seen, that there is a nonlinear relationship between the sensor signal and the hydrogen peroxide concentration in case of the measurement 1 . On the other hand, a linear relationship can be observed in case of measurement 2 , while during both measurements the sensor signal is decreasing with increasing hydrogen peroxide concentration. This seems to be apparently in contradiction to the expectation that $\mathrm{n}$-type metal-oxide-semiconductor gas sensors, like the $\mathrm{SnO}_{2}$ sensor used in this work, respond with an increase of the sensor resistance in presence of oxidizing gases, like hydrogen peroxide. However, to explain the effect of hydrogen peroxide on the metal oxide surface, several mechanisms have to be considered. Thereby, it is assumed that the change in sensor signal relies not merely on a direct influence of the hydrogen peroxide on the metal oxide surface, but is predominantly attributed to the effect of decomposition products of hydrogen peroxide at the sensor surface.

The decomposition of hydrogen peroxide vapour at ceramic oxide surfaces has been the subject of few earlier investigations [24]. At high temperatures $\left(>400^{\circ} \mathrm{C}\right)$, which is the case at the hot sensor surface, the decomposition of hydrogen peroxide is thought to be initiated by $\mathrm{O}-\mathrm{O}$ cleavage to give two $\mathrm{OH}^{*}$ radicals followed by two simple reactions.

$$
\begin{aligned}
& \mathrm{H}_{2} \mathrm{O}_{2} \rightarrow 2 \mathrm{OH}^{\bullet}, \\
& \mathrm{OH}^{\bullet}+\mathrm{H}_{2} \mathrm{O}_{2} \rightarrow \mathrm{HO}_{2}+\mathrm{H}_{2} \mathrm{O}, \\
& 2 \mathrm{HO}_{2} \rightarrow \mathrm{H}_{2} \mathrm{O}_{2}+\mathrm{O}_{2} .
\end{aligned}
$$


The stoichiometric relationship gives half an oxygen molecule for every hydrogen peroxide molecule decomposed.

$$
\mathrm{H}_{2} \mathrm{O}_{2} \rightarrow \mathrm{H}_{2} \mathrm{O}+1 / 2 \mathrm{O}_{2}
$$

The so formed $\mathrm{OH}^{\cdot}$ radicals on the oxide surface are of transitory nature, but may act as electron donors and directly increase the conductivity of the metal oxide, in this case tin oxide. This model explains the strikingly decrease of the sensor signal in presence of hydrogen peroxide as shown in Fig. 1. A similar effect can be observed in the presence of water. When chemisorbed on the tin-oxide surface, water molecules dissociate into hydroxyl species, which again act as electron donors $[25,26]$. That means hydrogen peroxide and water will contribute to the change in conductivity of the tin oxide in the same way. From the values obtained during measurement 2 it can be seen, that the formation of free hydroxyl radicals being descended from the hydrogen peroxide is predominant for the change in the sensor's conductivity, as the sensor signal is lower for higher hydrogen peroxide concentrations (compare also Table 2). The effect of humidity may be observed comparing the obtained values of measurement 1 and 2 . The sensor signal at $2.2 \% \mathrm{v} / \mathrm{v} \mathrm{H}_{2} \mathrm{O}_{2}$ derived from measurement 2 is lower compared to that of measurement 1 , which comes as a result of the higher level of humidity during measurement 2 but similar concentrations of hydrogen peroxide (compare also Table 2).

3.3 SO-A0-250 The resulting sensor response of the solid-electrolyte gas sensor SO-A0-250 during measurements 1 and 2 in hydrogen peroxide atmosphere is shown in Fig. 2. The measured oxygen concentration is plotted against the assumed hydrogen peroxide concentration calculated from the mixer flow ratios. The sensor shows a linear behaviour as a function of the hydrogen peroxide

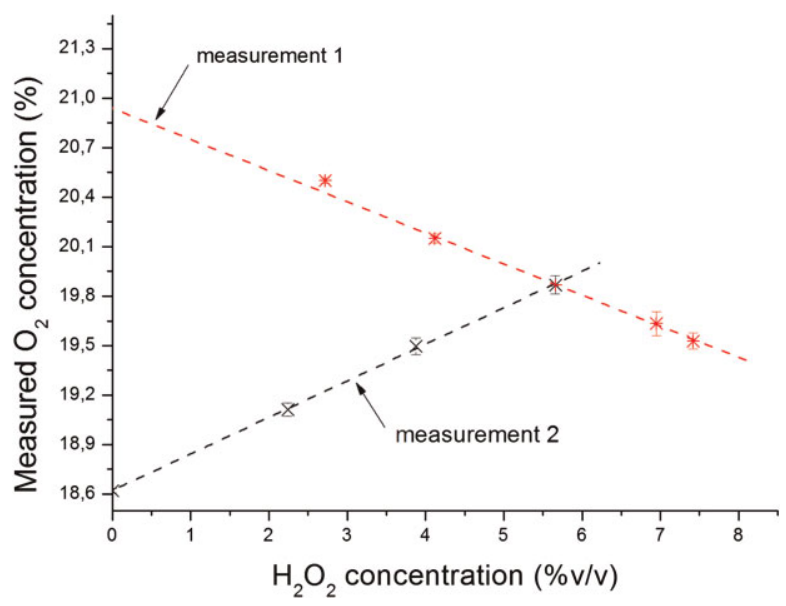

Figure 2 (online colour at: www.pss-a.com) Measured oxygen concentration of the amperometric solid-electrolyte sensor SOA0-250 at different concentrations of hydrogen peroxide operated in temperature-controlled mode at $533{ }^{\circ} \mathrm{C}$. concentration. The sensor signal is decreasing towards higher concentrations of $\mathrm{H}_{2} \mathrm{O}_{2}$ during measurement 1 , while it is increasing towards higher concentrations during measurement 2. To explain this behaviour, one must consider that the solid-electrolyte gas sensor is sensitive in particular to oxygen and therefore the sensor signal depends on the oxygen concentration in the medium. The progressive increase of the amount of hydrogen peroxide solution fed to the carrier gas stream leads to a decrease in the relative oxygen content of the gas stream, which explains the declining sensor response in case of measurement 1 . On the other hand, the amount of dosed hydrogen peroxide solution was kept constant during measurement 2, while the dilution was varied from lower to higher concentrations in order to obtain rising hydrogen peroxide concentrations in gaseous phase. In this case, the relative oxygen content of the gas stream is considered to be constant, while the sensor signal is increasing with increasing hydrogen peroxide concentrations. The increase in sensor response observed during measurement 2 may be explained due to a supplementary source of oxygen, which is related to the heterogeneous decomposition of hydrogen peroxide into water and oxygen according to Eq. (5) at either the heater surface or even at the surface of the sensor itself, which is operated at a temperature of approximately $500{ }^{\circ} \mathrm{C}$. It can be considered that the solidelectrolyte sensor exhibits partial sensitivity towards hydrogen peroxide.

3.4 Microbiological tests The microbiological tests have been carried out as described in Section 2.3, each parameter with a repetition of five samples. The resulting average log-rates of the microbiological measurements 1 and 2 are shown in Fig. 3, plotted against the particular hydrogen peroxide concentration.

The results obtained at $0 \% \mathrm{v} / \mathrm{v} \mathrm{H}_{2} \mathrm{O}_{2}$ were taken in the hot gas stream at $270{ }^{\circ} \mathrm{C}$, in absence of hydrogen peroxide. The exposition to hot air for $500 \mathrm{~ms}$ causes only a minimal

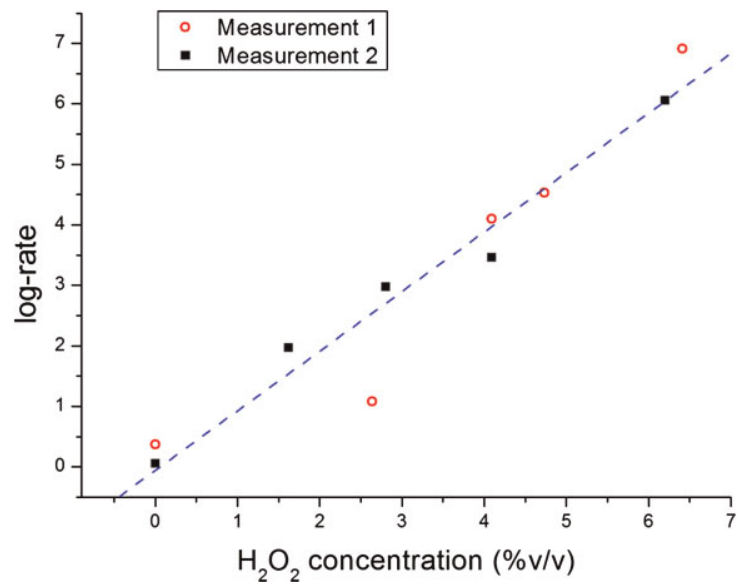

Figure 3 (online colour at: www.pss-a.com) Achieved logarithmic reduction rates of $B$. subtilis at $270^{\circ} \mathrm{C}$ as a function of the hydrogen peroxide concentration. 
microbiological reduction of viable spores. The achieved log-rate was in both cases below 0.5. In presence of hydrogen peroxide, the logarithmic reduction rate is significantly increased. For instance, at a concentration of $1.6 \% \mathrm{v} / \mathrm{v} \mathrm{H}_{2} \mathrm{O}_{2}$ during the microbiological measurement 2 the number of viable spores was reduced by two decades during an exposition time of $500 \mathrm{~ms}$. For all further samples from the microbiological measurements 1 and 2, the log-rate is increasing with increasing hydrogen peroxide concentration, except for the sample at $2.6 \% \mathrm{v} / \mathrm{v} \mathrm{H}_{2} \mathrm{O}_{2}$ from the microbiological measurement 1. At the highest $\mathrm{H}_{2} \mathrm{O}_{2}$ concentration of $6.4 \% \mathrm{v} / \mathrm{v}$ the maximum log-rate of 6.9 , which means that no viable spores remained, was obtained. Therefore, it was not considered necessary to further increase the hydrogen peroxide concentration.

It seems that the inactivation of B. subtilis spores is independent from the level of humidity in the sterilization medium. The logarithmic reduction rate of $B$. subtilis by hydrogen peroxide vapour at a gas temperature of $270{ }^{\circ} \mathrm{C}$ can be described as a linear function of the hydrogen peroxide concentration according to the following formula:

$$
\log \text {-rate }=-0.06+0.97(\% \mathrm{v} / \mathrm{v})^{-1} * \mathrm{C}_{[}\left[\mathrm{H}_{2} \mathrm{O}_{2}\right](\% \mathrm{v} / \mathrm{v}) .
$$

3.5 Correlation In order to realize a sensor system for the evaluation of aseptic processes employing hydrogen peroxide vapour, a correlation between sensor data and the results of the microbiological tests has to be established.

Based on the known sensor behaviour from the calibration measurements and the relationship found between the hydrogen peroxide concentration and the logarithmic reduction rate, which is described in Eq. (6), the log-rate may be described as a function of the sensor output of the two sensors in test. Furthermore, additional sensor data and log-rates for all possible combinations of dosed amount of hydrogen peroxide solution between 250 and $1100 \mu \mathrm{l} / \mathrm{s}$ as described in Table 1 and respective values of hydrogen peroxide dilutions between 15 and $50 \% \mathrm{w} / \mathrm{w}$ listed in Table 4 have been approximated via the characteristics found during sensor calibration and microbiological test, respectively. From the obtained data an extrapolation was drawn for other possible variations of the gas composition, regarding the hydrogen peroxide concentration and level of humidity. The obtained data are representative for a gas flow of $10 \mathrm{~m}^{3} / \mathrm{h}$ and a gas temperature of $270{ }^{\circ} \mathrm{C}$. The resulting graph is shown in Fig. 4. Thereby, the resulting microbiological log-rate is represented as coloured scale as a function of the sensor signals of the TGS 816 and SO-A0250 , respectively.

Hereby, the logarithmic reduction rate of B. subtilis spores is represented as a function of the sensor output and diagrammed as coloured scale. From the graph shown in Fig. 4, the microbial reduction may be estimated by means of a given combination of sensor signals. The level of reliability

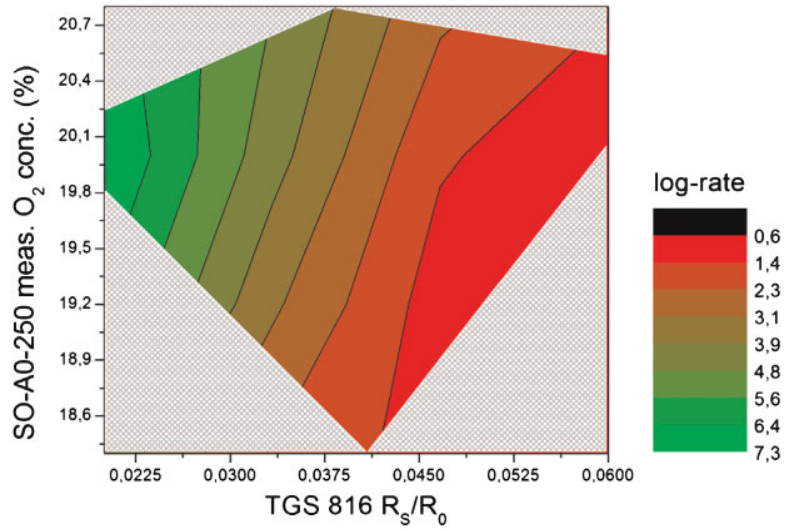

Figure 4 (online colour at: www.pss-a.com) Extrapolated data set of the logarithmic reduction rate (log-rate) of B. subtilis spores as a function of the output values of two commercially available gas sensors (TGS 816 and SO-A0-250).

of the approximation derives from the standard deviation of the sensor calibration and the averaging of the log-rate. Based on the established correlation, the resulting log-rate of an unknown gas composition may be estimated by reference to the sensor signals with an accuracy of \pm 0.85 log-rates.

4 Conclusion Two types of commercially available gas sensors have been investigated on cross-sensitivity towards hydrogen peroxide vapour. Additionaly, microbiological CRTs of B. subtilis spores have been performed, in order to determine the sterilization effect of hydrogen peroxide vapour. The calibration of the sensor system and the microbiological tests have been carried at a gas flow of $10 \mathrm{~m}^{3} / \mathrm{h}$ and a gas temperature of $270^{\circ} \mathrm{C}$. A correlation between the sensor output and the results of the microbiological tests has been established. Based on this correlation, the microbiological reduction rate of an unknown gas composition may be estimated by means of the sensor output with an accuracy of $\pm 0.85 \log$-rates. The developed multi-sensor system is suitable for in-line and real-time analysis of the sterilization process.

In further studies, the field of parameters shall be extended by other influencing factors of the sterilization process, namely the temperature and gas flow. Also, the number and type of gas sensors shall be increased in order to obtain more detailed chemical images of the medium to be analyzed. The increase in number of sensors and variables requires the introduction of a multivariate analysis, like Fuzzy Logic, Artificial Neural Networks (ANN) or Principal Component Analysis (PCA) in order to handle the captured information. The instrumental systems based on arrays of low-selective/cross-reactive sensors that are used to form chemical images of gaseous multi-component media with further classification and identification using the image recognition techniques has been named the Electronic Nose' [27]. 


\section{References}

[1] S. D. Holdsworth, Aseptic Processing and Packaging of Food Products (Elsevier Science Publishing, New York, 1992), p. 428.

[2] G. McDonnell and D. Russell, Clin. Microbiol. Rev. 12, 147 (1999).

[3] R. A. Heckert, M. Best, L. T. Jordan, D. L. Eddington, and W. G. Sterritt, Appl. Environ. Microbiol. 63, 3916 (1997).

[4] M. Kokubo, T. Inoue, and J. Akers, PDA J. Pharm. Sci. Technol. 52, 228 (1998).

[5] R. Toniolo, P. Geatti, G. Bontempelli, and G. Schiavon, J. Electroanal. Chem. 514, 123 (2001).

[6] N. A. Klapes and D. Vesley, Appl. Environ. Microbiol. 56, 503 (1990).

[7] G. S. Graham, J. R. Rickloff, and J. P. Dalmasso, in: Proceedings of the International Congress of Parenteral Drug Association (Basel, Switzerland, 1992), pp. 32-51.

[8] C. Hultman, A. Hill, and G. McDonnell, Pharm. Eng. 27, 22 (2006).

[9] V. Sigwart and A. Stärk, PDA J. Pharm. Sci. Technol. 57, 3 (2003).

[10] K. Imai, S. Watanabe, Y. Oshima, M. Kokubo, and J. Akers, Pharm. Eng. 26, 96 (2006).

[11] B. Unger-Bimezok, V. Kottke, C. Hertel, and J. Rauschnabel, J. Pharm. Innov. 3, 123 (2008).

[12] M. D. Labas, C. S. Zalazar, R. J. Brandi, and A. E. Cassano, Biochem. Eng. J. 38, 78 (2008).

[13] R. Amman, in: Code of Practice - Testing the Effectiveness of Aseptic Plants Fitted with Packaging Sterilization Devices, VDMA-Documents - Food Processing Machinery and Packaging Machinery, Vol. 6 (VDMA, Frankfurt, 2003).
[14] G. Moruzzi, W. E. Garthright, and J. D. Floros, Food Control 11, 57 (2000).

[15] I. Taizo, A. Sinichi, and K. Kawamura, PDA J. Pharm. Sci. Technol. 52, 12 (1998).

[16] N. Näther, H. Henkel, A. Schneider, and M. J. Schöning, Phys. Status Solidi A 206, 449 (2009).

[17] N. Näther, R. Emmerich, J. Berger, P. Friedrich, H. Henkel, A. Schneider, and M. J. Schöning, Sensors 6, 308 (2006).

[18] N. Näther, L. M. Juárez, R. Emmerich, J. Berger, P. Friedrich, and M. J. Schöning, Mater. Res. Soc. Symp. Proc. 951, 0951E12-03 (2007).

[19] S. Reisert, H. Henkel, A. Schneider, D. Schäfer, P. Friedrich, J. Berger, and M. J. Schöning, Phys. Status Solidi A 207, 913 (2010).

[20] S. Corveleyn, G. M. Vandenbossche, and J. P. Remon, Pharm. Res. 14, 294 (1997).

[21] J. Wang and D. A. Mondiek, Sterilant monitoring assembly and apparatus and method using same, US Patent, US 006517775 B1 (1999).

[22] B. A. Smopok and I. V. Kruglenko, Thin Solid Films 418, 21 (2002).

[23] W. Göpel, Sens. Actuators B 52, 125 (1998).

[24] A. Hiroki and J. A. LaVerne, J. Phys. Chem. B 109, 3364 (2005).

[25] D. Kohl, Sens. Actuators B 24/25, 491 (1995).

[26] P. D. Skafidas, D. S. Vlachos, and J. N. Avaritsiotis, Sens. Actuators B 18/19, 724 (1994).

[27] T. C. Pearce, S. S. Schiffmann, H. T. Nagel, and J. Gardner, Handbook of Machine Olfaction (Wiley-VCH, Weinheim, 2003), p. 79. 